\title{
THE SPECTRUM OF SKEW ROOM SQUARES
}

\author{
D. R. STINSON
}

(Received 12 February 1981)

Communicated by W. D. Wallis

\begin{abstract}
We give a short proof that skew Room squares exist for all odd sides $s$ exceeding 5 . 1980 Mathematics subject classification (Amer. Math. Soc.): 05 B 30.
\end{abstract}

\section{Introduction}

A Room square of side $s$ is a square array $R$ of side $s$, satisfying:

(1) each cell of $R$ is either empty or contains a 2-subset of a set $S$ of size $s+1$,

(2) each symbol occurs exactly once in each row and each column of $R$,

(3) every 2-subset of $S$ occurs in a unique cell of $R$.

The existence question for Room squares has been solved. The following is shown by Mullin and Wallis in [4].

THEOREM 1.1. A Room square of side $s$ exists if and only if $s$ is an odd positive integer other than 3 or 5.

A Room square $R$, on symbol set $S$, is said to be standardized with respect to $\infty \in S$ provided that the rows and columns of $R$ have been permuted so that $\infty$ occurs in the cells on the main diagonal of $R$. We will index the rows and

Copyright Australian Mathematical Society 1981 
columns of such a standardized Room square by $S \backslash\{\infty\}$, so that $\{\infty, x\}$ occurs in cell $(x, x)$, for all $x \in S \backslash\{\infty\}$.

A standardized Room square is skew provided that, of any two cells $(i, j)$ and $(j, i)$ with $i \neq j$, precisely one is empty. Skew Room squares have been studied extensively: see the list of references.

We show here that a skew Room square of side $s$ exists if and only if $s$ is an odd positive integer other than 3 or 5 , that is, skew Room squares exist for precisely the same set of sides as Room squares.

\section{Frames and Room squares}

Let $S$ be a set of size $s$, and partition $S=\cup_{j=1}^{n} S_{j}$.

An $\left\{S_{1}, \ldots, S_{n}\right\}$-frame is a square array $F$ of side $s$, having rows and columns indexed by $S$, which satisfies:

(1) each cell is either empty or contains a 2-subset of $S$,

(2) the subsquares $S_{j}^{2}$ of $F$ are empty, for $1 \leqslant j \leqslant n$,

(3) row (or column) $x$ contains precisely the symbols $S \backslash S_{j}$, where $x \in S_{j}$,

(4) the 2-subsets occurring in $F$ are precisely those $\{x, y\}$ such that $(x, y) \in S^{2} \backslash \cup_{j=1}^{n} S_{j}^{2}$.

A frame is skew provided that for all $(i, k) \in S^{2} \backslash \cup_{j=1}^{n} S_{j}^{2}$, exactly one of cell $(i, k)$ or $(k, i)$ is empty.

A Room square of side $s$ (standardized with respect to some element $\infty \notin S$ ) can be constrtucted from an $\{\{s\}: s \in S\}$-frame by placing $\{\infty, x\}$ in cell $(x, x)$, for all $x \in S$. Similarly, a skew Room square can be constructed from a skew frame of that type.

The study of Room squares predates that of frames, of course. However, the above construction indicates the fact that frames are a generalization of Room squares. The study of frames seems to be of interest in its own right, as well for applications in the construction of Room squares and Howell designs. The reader is referred to [1], [2], [5], [6], [10], [12], [15],[16], [17], and [18].

We make use of frames as follows.

Lemma 2.1. Suppose a skew $\left\{S_{1}, \ldots, S_{n}\right\}$-frame exists, and suppose also that skew Room squares of side $\left|S_{i}\right|+1$ exist, for $1 \leqslant i \leqslant n$. Then a skew Room square of side $1+\sum_{i=1}^{n}\left|S_{i}\right|$ exists.

Proof. See [16, Theorem 3.1] 
We will describe a construction for frames which uses group-divisible designs (GDDs). A GDD is a triple ( $X, \mathcal{G}, \mathscr{Q}$ ) where $X$ is a set (of points), $\mathcal{G}$ is a set of subsets of $X$ (called blocks), such that every 2-subset of $X$ not contained in a group is contained in a unique block, and such that a group and a block contain at most one common point. A weighting of a GDD is a map $w: X \rightarrow \mathbf{Z}^{+} \cup\{0\}$. The following construction is a generalization of [16, Theorem 2.1], where all weights are equal. The reader will note the similarity between this construction and Wilson's fundamental construction for GDDs [20].

Construction 2.2. Suppose $(X, \mathcal{G}, \mathfrak{Q})$ is a GDD and $w$ is a weighting. For every $x \in X$, let $S_{x}$ be a set of size $w(x)$. Suppose these $S_{x}$ 's are disjoint, and for any $T \subseteq S$, define $S_{T}=\cup_{x \in T} S_{x}$. For every block $A \in \mathbb{Q}$, suppose $F_{A}$ is a skew $\left\{S_{x}: x \in A\right\}$-frame. Let $F$ be the array obtained by superimposing all these $F_{A}$ 's. Then $F$ is a skew $\left\{S_{G}: G \in \mathcal{G}\right\}$-frame.

In the next section, we derive a corollary to the above construction which will enable us to close the spectrum of skew Room squares, given an initial segment.

We close this section by indicating four frames we will use in the next section. We say that an $\left\{S_{1}, \ldots, S_{n}\right\}$-frame has type $t_{1}^{u_{1}} \cdots t_{k}^{u_{k}}$ if there are $u_{i} S_{j}$ 's of size $t_{i}$, for $1 \leqslant i \leqslant k$.

LEMMA 2.3. There exist skew frames of type $4^{4}, 4^{4} 2^{1}, 4^{5}$, and $4^{4} 6^{1}$.

Proof. See [17].

\section{The spectrum}

LEMMA 3.1. Suppose there exist three MOLS (mutually orthogonal Latin squares) of order $m$, and skew Room squares of sides $4 m+1$ and $2 t+1$, where $0 \leqslant t \leqslant 3 m$. Then a skew Room square of side $16 m+2 t+1$ exists.

Proof. Since three MOLS of order $m$ exist, there is a $\operatorname{GDD}(X, \mathcal{G}, \mathbb{Q})$ with five groups of size $m$ and $m^{2}$ blocks of size 5 .

Define a weighting $w: X \rightarrow\{0,2,4,6\}$ by setting $w(x)=4$ if $x \in X \backslash G_{5}$, and defining $w(x)$ for $x \in G_{5}$ so that $\Sigma_{x \in G_{5}} w(x)=2 t$. 
Now apply Construction 2.2. A block $A$ requires one of the four frames of Lemma 2.3, and the result is a skew frame of type $4 m^{4} 2 t^{1}$. Apply Lemma 2.1 to obtain a skew Room square of side $16 m+2 t+1$.

Denote SRS $=\{s \geqslant 7:$ a skew Room square of side $s$ exists $\}$.

Lemma 3.2 Suppose $\{7,9, \ldots, 117\} \subseteq$ SRS. Then SRS $=\{s>7: s$ odd $\}$

Proof. The proof is by induction on $s$. Let $s \geqslant 119$ be odd, and write $s=16 m+2 t+1$ with $m$ odd and $3 \leqslant t \leqslant 18$. Then $m \geqslant 7$, so $t \leqslant 3 m$. Since $m$ is odd and exceeds 5, there exist three MOLS of order $m$ by [19]. Lemma 3.1 implies the result.

Thus we have only to construct skew Room squares of sides 7 through 117 . We will do this mainly by using results which appear in the literature.

Lemma 3.3. Suppose $q=2^{n} t+1$ is a prime power with $t$ odd, $t>1$. Then $q \in \mathrm{SRS}$.

Proof. Mullin and Nemeth [8].

Lemma 3.4. Suppose $s=16 t^{2}+1$. Then $s \in \mathrm{SRS}$.

Proof. Dinitz [4].

LEMMA 3.5 .

(i) If $\{u, v\} \subseteq \mathrm{SRS}$, then $u v \in \mathrm{SRS}$.

(ii) If $\{u, v\} \subseteq$ SRS and $v \neq 7$, then $u(v-1)+1 \in$ SRS.

(iii) If $v \in \mathrm{SRS}, v \neq 13$, and $u \equiv 1 \bmod 4$ is a prime power, then $u(v-1)+1$ $\in$ SRS.

Proof. For (i) and (ii), see [9]; for (iii), see [10].

The other small skew Room squares have been constructed by various methods, some of which are generalizations of the constructions of Lemma 3.5. In Table 1 below, we list sides from 7 to 117 which are not constructed by Lemmas 3.3 and 3.4, together with a reference describing their construction. 


\section{TABLE 1}

\begin{tabular}{|c|c|}
\hline Order & Construction \\
\hline 9 & Beaman and Wallis [3] \\
\hline 15 & Mullin, Schellenberg, Stinson and Vanstone [9] \\
\hline 21 & {$[9]$} \\
\hline 33 & Mullin and Wallis [13] \\
\hline 35 & [9] \\
\hline 39 & [9] \\
\hline 45 & [9] \\
\hline 51 & Lemma 3.5 (iii), $51=5(11-1)+1$ \\
\hline 55 & Lemma 3.5(iii), $55=9(7-1)+1$ \\
\hline 57 & Lemma 3.5(ii), $57=7(9-1)+1$ \\
\hline 63 & Lemma $3.5(i), 63=7 \cdot 9$ \\
\hline 69 & Stinson $[17]$ \\
\hline 75 & Anderson, Mullin and Stinson [1] \\
\hline 77 & Lemma $3.5(i), 77=7 \cdot 11$ \\
\hline 85 & Lemma 3.5 (ii), $85=7(13-1)+1$ \\
\hline 87 & Lemma $3.1, m=5, t=3$ \\
\hline 91 & Lemma $3.5(\mathrm{i}), 91=7 \cdot 13$ \\
\hline 93 & Stinson [16] \\
\hline 95 & Lemma $3.1, m=5, t=7$ \\
\hline 99 & Lemma $3.5(i), 99=9 \cdot 11$ \\
\hline 105 & Lemma 3.5(i), $105=7 \cdot 15$ \\
\hline 111 & Lemma 3.5(ii), $111=11(11-1)+1$ \\
\hline 115 & {$[1]$} \\
\hline 117 & Lemma $3.5(i), 117=9 \cdot 13$ \\
\hline
\end{tabular}

THEOREM 3.6. SRS $=\{s: s \geqslant 7$ is odd $\}$.

Proof. Lemmata 3.1-3.5 and Table 1.

\section{References}

[1] B. A. Anderson, R. C. Mullin, and D. R. Stinson, 'A few more skew Room squares', Utilitas Math., to appear.

[2] I. R. Beaman and W. D. Wallis, 'On skew Room squares', Proc. Fifth Australian Conf. on Combinatorial Mathematics (1977), 61-63.

[3] I. R. Beaman and W. D. Wallis, 'A skew Room square of side nine', Utilitas Math. 8 (1975), 382. 
[4] J. H. Dinitz (Ph.D. Thesis, Ohio State Univ., 1980).

[5] J. H. Dinitz and D. R. Stinson, 'The construction and uses of frames', Ars Combinatoria 10 (1980), to appear.

[6] J. H. Dinitz and D. R. Stinson, 'Further results on frames', Ars Combinatoria, to appear.

[7] R. C. Mullin, 'A generalization of the singular direct product with applications to skew Room squares', J. Combinatorial Theory Ser. A, to appear.

[8] R. C. Mullin and E. Nemeth, 'An existence theorem for Room squares', Canad. Math. Bull. 12 (1969), 493-497.

[9] R. C. Mullin, P. J. Schellenberg, D. R. Stinson, and S. A. Vanstone, 'Some results on the existence of squares', Annals of Discrete Math. 6 (1980), 257-274.

[10] R. C. Mullin, P. J. Schellenberg, S. A. Vanstone, and W. D. Wallis, "On the existence of frames', Discrete Math., to appear.

[11] R. C. Mullin, D. R. Stinson, and W. D. Wallis, 'Concerning the spectrum of skew Room squares', Ars Combinatora 6 (1987), 277-291.

[12] R. C. Mullin, D. R. Stinson, and W. D. Wallis, 'Skew squares of low order', Proc Eighth Manitoba Conf. on Numerical Mathematics and Computing, Winnipeg (1978), 413-434.

[13] R. C. Mullin and W. D. Wallis, 'Recent advances in complementary and skew Room squares', Proc. Fourth Southeastern Conf. on Combinatorics, Graph Theory and Computing, Boca Raton, Fla. (1973), 521-532.

[14] R. C. Mullin and W. D. Wallis, 'The existence of Room squares', Aequationes Math. 13 (1975), 1-7.

[15] D. R. Stinson, 'A skew Room square of order 129', Discrete Math., 31 (1980), 333-335.

[16] D. R. Stinson, 'Some results concerning frames, Room squares and subsquares', J. Austral. Math. Soc., to appear.

[17] D. R. Stinson, 'Some constructions for frames, Room squares, and subsquares', preprint.

[18] D. R. Stinson and W. D. Wallis, 'Some designs used in constructing skew Room squares', First Canada-France Combinatorial Colloquium, Montreal (1979), to appear.

[19] S. M. P. Wang and R. M. Wilson, A few more squares II, Proc. Ninth Southeastern Conf. on Combinatorics, Graph Theory and Computing, Boca Raton, Fla. (1978), 688 (abstract).

[20] R. M. Wilson, Constructions and uses of pairwise balanced designs (Math. Centre Tracts 55 (1974), 18-41).

\section{Department of Combinatorics and Optimization \\ University of Waterloo \\ Waterloo, Ontario \\ Canada}

